\title{
Encoding information using Laguerre Gaussian modes
}

Trichili, Abderrahmen, Dudley, Angela, Ben Salem, Amine, Ndagano, Bienvenu, Zghal, Mourad, et al.

Abderrahmen Trichili, Angela Dudley, Amine Ben Salem, Bienvenu Ndagano, Mourad Zghal, Andrew Forbes, "Encoding information using Laguerre Gaussian modes," Proc. SPIE 9581, Laser Beam Shaping XVI, 95810F (25 August 2015); doi: 10.1117/12.2189814

EDent: SPIE Optical Engineering + Applications, 2015, San Diego, California, United States 


\title{
Encoding Information using Laguerre Gaussian modes
}

\author{
Abderrahmen Trichili ${ }^{1}$, Angela Dudley ${ }^{2,3}$, Amine Ben Salem ${ }^{1}$, Bienvenu Ndagano ${ }^{3}$, Mourad \\ Zghal $^{1,4}$ and Andrew Forbes ${ }^{2,3 *}$ \\ ${ }^{1}$ University of Carthage, Engineering School of Communication of Tunis (Sup'Com), \\ GreS'Com Laboratory, Ghazala Technopark, 2083, Ariana, Tunisia \\ ${ }^{2}$ CSIR National Laser Centre, P.O. Box 395, Pretoria 0001, South Africa \\ ${ }^{3}$ School of Physics, University of the Witwatersrand, Johannesburg 2050, South Africa \\ 4Institut Mines-Télécom/Télécom SudParis, 9 rue Charles Fourier, 91011 Evry, France
}

\begin{abstract}
We experimentally demonstrate an information encoding protocol using the two degrees of freedom of Laguerre Gaussian modes having different radial and azimuthal components. A novel method, based on digital holography, for information encoding and decoding using different data transmission scenarios is presented. The effects of the atmospheric turbulence introduced in free space communication is discussed as well.
\end{abstract}

Keywords: Laguerre Gaussian modes, Digital Holography, Multiplexing, Free space communication

\section{INTRODUCTION}

Over the last few years, there has been considerable interest in mode division multiplexing (MDM) for data transmission in optical fibers and free space optical links. ${ }^{1}$ MDM is a promising technique to cope with an eventual capacity "crunch" in the near future. ${ }^{2}$ Using the spatial modes of light, the overall capacity of the communication systems increases by several orders of magnitude. ${ }^{3}$ Recent works have shown high transmission capacity using various spatial mode basis. Y. Chen et al. have demonstrated in [4] a $41.6 \mathrm{~Tb} / \mathrm{s}$ transmission capacity using 6 linearly polarised modes on dual polarisation over $74.17 \mathrm{~km}$ in a few mode fiber. In free space communication, J. Wang et al. in [5] have reported a bit rate of $2.56 \mathrm{~Tb} / \mathrm{s}$ and a spectral efficiency of 95.7 $\mathrm{b} / \mathrm{s} / \mathrm{Hz}$ using 4 orbital angular momentum (OAM) modes. Moreover, a $400 \mathrm{Gbit} / \mathrm{s}$ transmission capacity over a free space optical link and using 4 Laguerre Gaussian (LG) modes with multiple OAM and the same higher radial order has been achieved. ${ }^{6}$ The practical implementation of MDM systems requires enabling new mode multiplexing and demultiplexing techniques. One technique is to use spatial light modulators (SLM) to generate and detect various high order modes. ${ }^{7-9}$

In this contribution, we focus on further increasing the number of multiplexed spatial modes to achieve higher transmission capacity using computer generated holograms. Here we show a novel technique for information encoding using Laguerre Gaussian (LG) modes with different azimuthal and radial indices offering two degrees of freedom compared to the previous communication techniques. ${ }^{10-12}$ Throughout this paper we consider only the case of free space propagation of a light beam to illustrate the concept, we implement our technique to encode and transmit gray-scale and RGB images on light beams. We perform a real time modal decomposition without prior knowledge on the incoming field and with a single digital hologram. We show the versatility of our technique by introducing atmospheric turbulence and how we are able to recover the sent information with high fidelity.

Further author information: (Send correspondence to A. Forbes)

A. Forbes: E-mail:Andrew.Forbes@wits.ac.za

Laser Beam Shaping XVI, edited by Andrew Forbes, Todd E. Lizotte, Proc. of SPIE Vol. 9581 95810F · @ 2015 SPIE · CCC code: 0277-786X/15/\$18 · doi: 10.1117/12.2189814

Proc. of SPIE Vol. $958195810 \mathrm{~F}-1$ 


\section{CONCEPT AND EXPERIMENTAL SETUP}

\subsection{Theory}

The Laguerre Gaussian beams are the solutions of the Helmholtz equation and form a complete orthonormal basis and each beam is characterized by an azimuthal index $\ell$ and a radial index $p .{ }^{13}$ In cylindrical coordinates, the LG modes are described by ${ }^{14}$

$E_{(p, \ell)}^{L G}(r, \phi, z)=\frac{1}{w(z)} \sqrt{\frac{2 p !}{\pi(|\ell|+p) !}} \exp (i(2 p+|\ell|+1) \Phi(z)) \times\left(\frac{2 r^{2}}{w^{2}(z)}\right) L_{p}^{|\ell|}\left(\frac{r \sqrt{2}}{w(z)}\right)^{|\ell|} \exp \left(-\frac{i k r^{2}}{2 R(z)}-\frac{-r^{2}}{w^{2}(z)}+i l \phi\right)$

where $\Phi(z)$ represents the Gouy phase, $w(z)$ is the beam spot size parameter and $R(z)$ is the radius of the beam curvature and $L_{p}^{|\ell|}$ are the Laguerre polynomials.

The main idea of the present work is to encode information using LG modes and send them from a transceiver to a receiver. As a proof of concept, the information used are gray-scale and coloured images. As shown in the concept in Fig. 1, each pixel of a gray-scale image corresponds to a particular LG mode, the mode is propagated and then identified at the receiver with a single measurement and once all the pixels are transferred, the initial image is reconstructed. For an RGB image, each pixel is composed of three different colours: red, green and blue. Thus, each colour is encoded with a beam and the beams are multiplexed together and transmitted in one beam. Upon arrival to the destination, all the three modes are identified in the same measurement procedure and the initial colour value of the pixel is recovered.

Transceiver

(a)

(b)

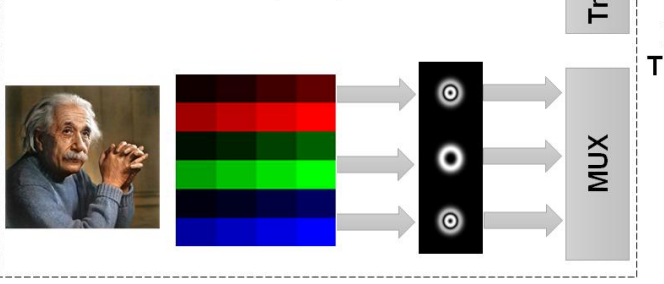

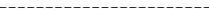

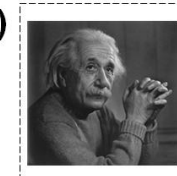

(b)
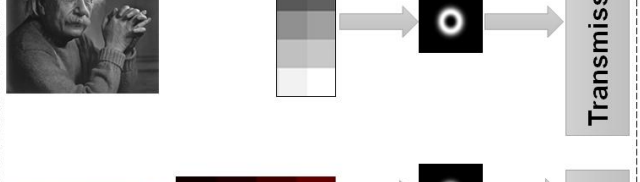

Free space

Transmission

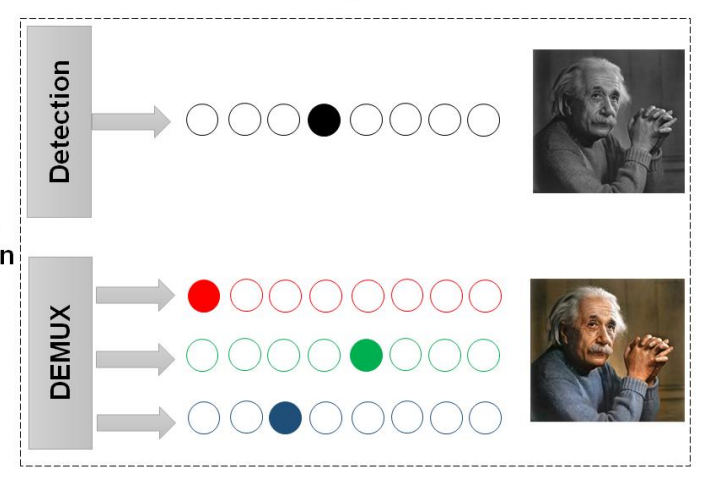

Figure 1. Concept of the proposed information encoding using LG modes. For a gray-scale image (a) each pixel value in the gray-scale corresponds to a particular mode wich is then propagated as a single beam through free space and identified in a single measurement at the receiver. For an RGB image (b) each pixel is encoded using three different modes and then propagated at the same time to a destination where the modes are demultipled and each colour is recoverd independently and then all of them are added together in order to recover the initial image pixel by pixel.

We are able to identify with a single measurement which mode is present in the receiver amongst the modes applied in the protocol. To apply such a demultiplexing method, we use digital holograms. Mathematically, the demultiplexing hologram is given by

$$
t_{D E M U X}=\sum E_{(p, \ell)}^{L G}(r, \phi, z)
$$

which consists of a superposition of all the protocol modes. An inner product measurement is performed optically, with the same idea in Ref [15] by considering the signal at the origin of the focal plane of the lens. The resulting signal at the receiver can be numerically calculated as

$$
I=\mathcal{F}\left(E_{(p, \ell)}^{L G}(r, \phi, z)\right) \otimes \mathcal{F}\left(t_{D E M U X}\right)
$$


where $I$ refers to the output signal and $\mathcal{F}$ represents the Fourier transform. $\otimes$ denotes the convolution process and $E_{(p, \ell)}^{L G}(r, \phi, z)$ is the LG mode previously defined in Eq. 1. Eq. 3 can then be expressed as

$$
I=\frac{\exp (i 2 k f)}{i \lambda f} \int_{0}^{\infty} \int_{0}^{2 \pi} t_{D E M U X} E_{(p, \ell)}^{L G}(r, \phi, z) r \mathrm{~d} r \mathrm{~d} \Phi
$$

where $f$ is the focal length of the lens used in the decomposition, $\lambda$ is the wavelength and $k$ is the wavenumber. The mode corresponds to a bright spot at the center of the output detector. If there is a mismatch between the incoming mode and the mode in the receiver the central spot will itself become a small ring with a low intensity in the center, which will cause a negligible signal on the detector. Note that each mode has a pre-defined position on the detector.

\subsection{Experimental Setup}

The experimental setup for information encoding using LG modes is shown in Fig. 2. An expanded HeNe laser beam was directed onto the liquid crystal display (LCD) at the first spatial light modulator (HoloEye Pluto-VIS with a $1920 \times 1080$ pixels of pitch $8 \mu \mathrm{m}$ and calibrated for a $2 \pi$ phase shift at $\lambda \sim 633 \mathrm{~nm}$ ) where the information was encoded in the LG modes. The resulting image was filtered using a $4 f$ imaging system and then imaged onto $\mathrm{SLM}_{2}$. The LCD of $\mathrm{SLM}_{2}$ was programmed with the multimode, demultiplexing hologram and the resulting signal was detected by a CCD camera placed after the Fourier transforming lens $\mathrm{L}_{5}$. The generation of the digital holograms in both SLMs was done using an amplitude modulation technique. ${ }^{16}$

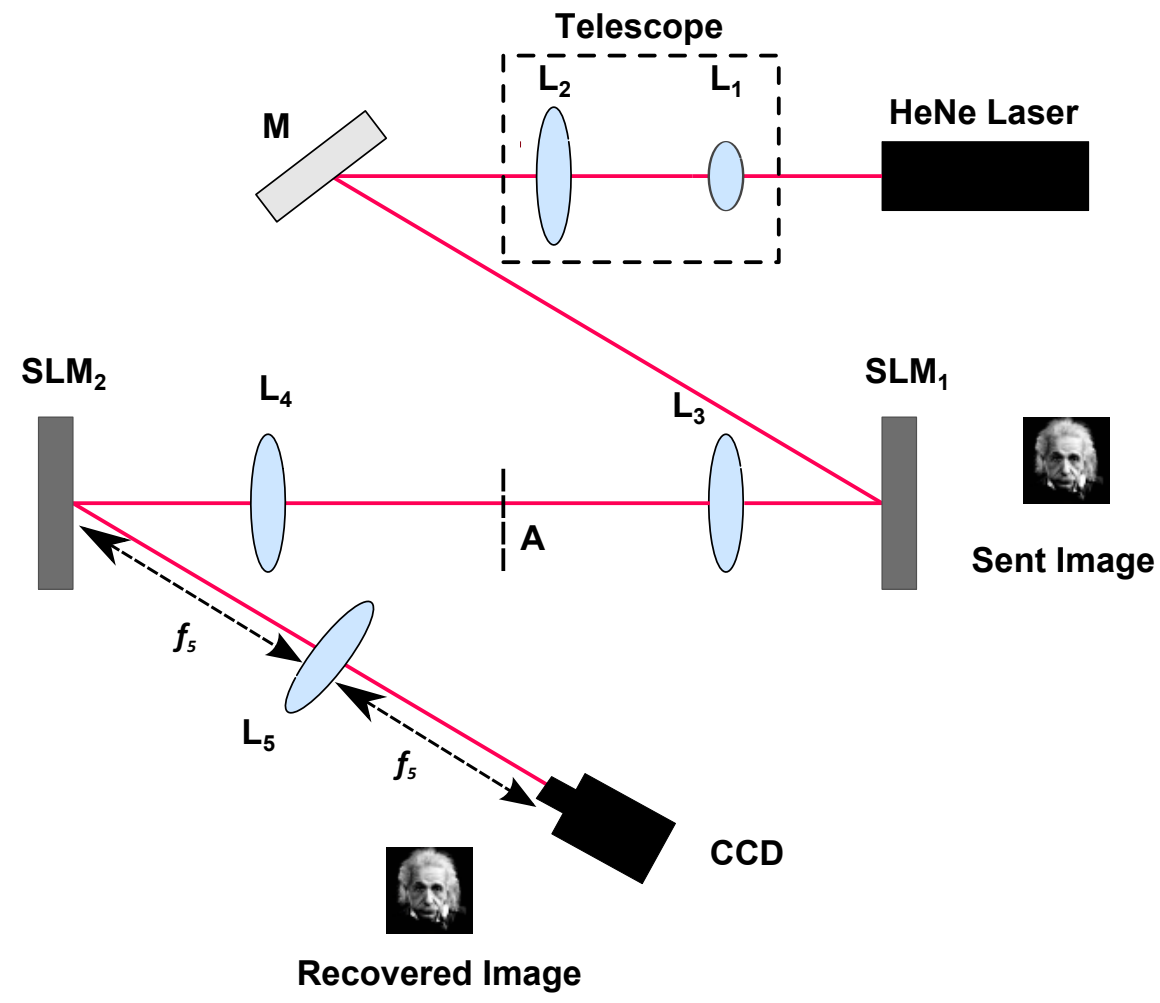

Figure 2. Schematic of the experimental setup for information encoding using Laguerre Gaussian modes. Lenses $\mathrm{L}_{1}, \mathrm{~L}_{2}$, $\mathrm{L}_{3}, \mathrm{~L}_{4}$ and $\mathrm{L}_{5}$ have focal lengths $f_{1}=50 \mathrm{~mm}, f_{2}=500 \mathrm{~mm}, f_{3}=500 \mathrm{~mm}, f_{4}=500 \mathrm{~mm}$ and $f_{5}=150 \mathrm{~mm}$, respectively. $\mathrm{A}$ is a filtering aperture. $\mathrm{SLM}_{1}$ and $\mathrm{SLM}_{2}$ denote the spatial light modulators and $\mathrm{M}$ represents a mirror. The detector is a CCD camera.

We first program our input gray-scale image on our system. Since the SLM can be dynamically addressed, the hologram is generated on $\mathrm{SLM}_{1}$ according to the value of the first pixel of the input image and then a beam 
is transmitted to the second SLM which is programmed with the multimode demultiplexing hologram. The incoming mode is identified and the pixel value is recorded. The processing is continuously done pixel by pixel and here we assume that the size of the image and the position of each transmitted pixel are known by the receiver.

As explained in section 2.1, when the image is RGB, three modes are multiplexed and a multimode hologram is programmed on $\mathrm{SLM}_{1}$ resulting in a single beam (containing the pixel information) being transmitted to $\mathrm{SLM}_{2}$ where a multimode demultiplexing hologram is used to identify all the colours. The pixel information is recorded and the image is recovered pixel by pixel.

\section{EXPERIMENTAL RESULTS AND DISCUSSION}

Using the information encoding technique described above, the results are depicted in Fig. 3. We were able to transmit and recover gray-scale images with 30 different gray-scale values and RGB images with 10 different RGB values.
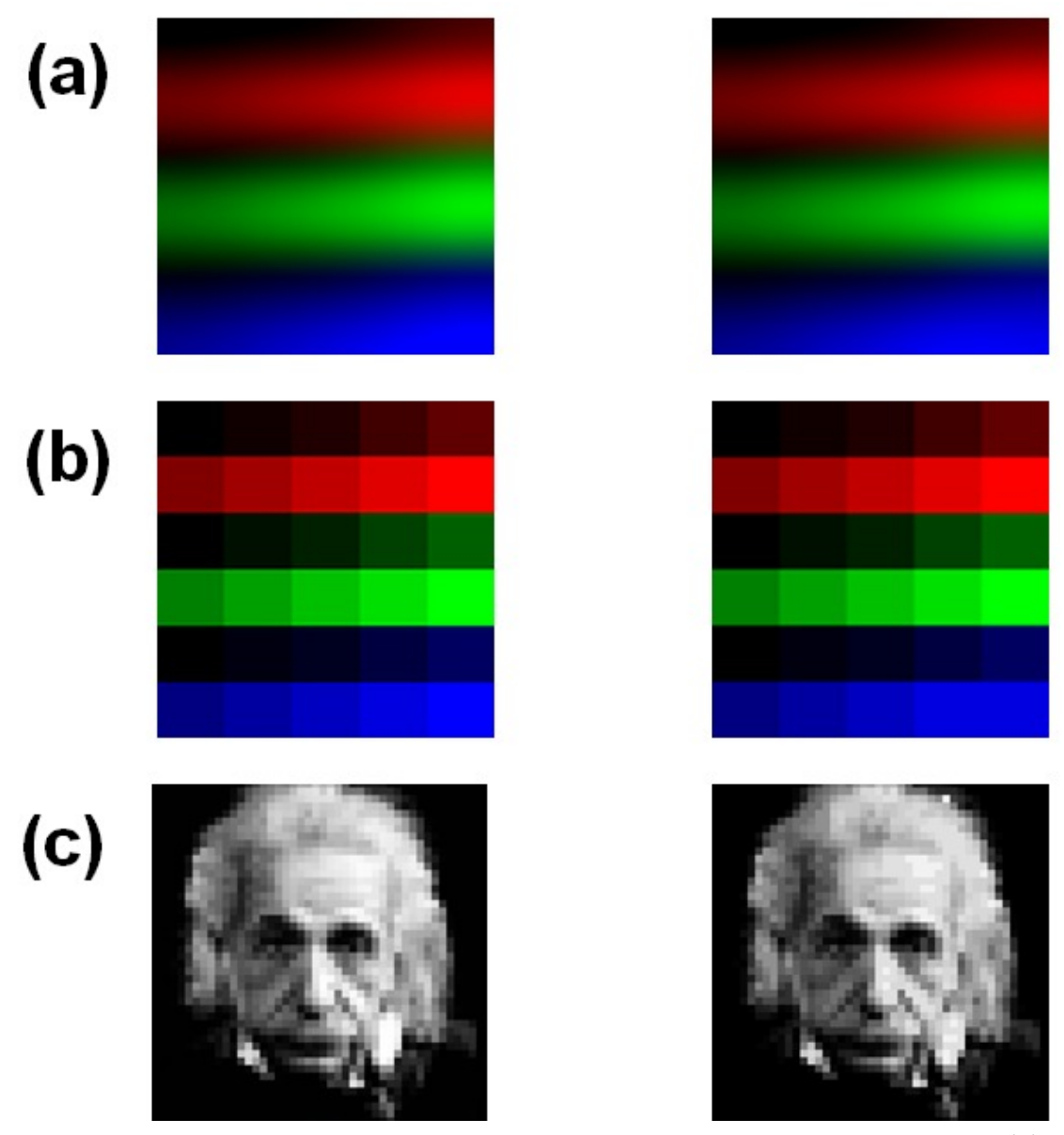

Figure 3. Examples of the images transmitted using the proposed information encoding method (a) and (b) are 10 values RGB images and (c) 30 gray-scale images. On the left the original images and on the right the recovered images.

To illustrate the robustness of the proposed method, we slightly changed the setup described in Fig. 2 and we placed after the first SLM a turbulence plate encoded with the Kolmogrov turbulence model. The turbulence strength is measured by means of the Strehl ratio (SR). ${ }^{17}$ We recall that when the SR is moving towards 0 the turbulence strength increases. We make use of four different LG modes with the following indices: $(p=1, \ell=2)$, $(p=1, \ell=-2),(p=0, \ell=5)$ and $(p=0, \ell=-5)$ to encode a four gray-scale image. 


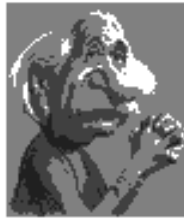

(a)

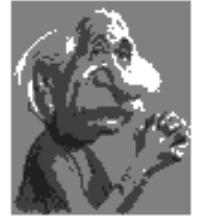

(b)

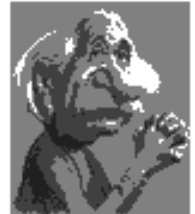

(c)

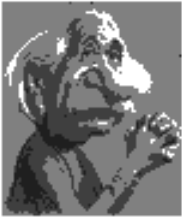

(d)

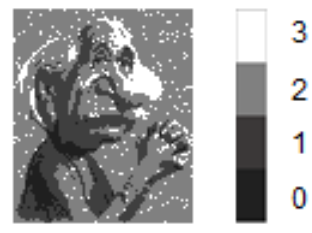

(e)

Figure 4. (a) Original 4 gray-scale Albert Einstein image. Received image for (b) $\mathrm{SR}=1$, (c) $\mathrm{SR}=0.8$, (d) $\mathrm{SR}=0.6$ and (e) $\mathrm{SR}=0.4$.

The initial image and corresponding reconstructed images (Albert Einstein images, $89 \times 75$ pixels) are depicted in Fig. 4. For $\mathrm{SR}=1$ and $\mathrm{SR}=0.8$, we find that we are able to recover the initial image with $100 \%$ fidelity as depicted in Fig. 4 (b) and Fig. 4 (c). However, in Fig. 4 (d) for a SR=0.6 the average error transmission rate based on the sent and received measured pixel intensities is found to be very low and has a value around $4.5 \times 10^{-4} \%$. For an intense turbulent medium where $\mathrm{SR}=0.4$, the image was distorted with an average transmission error of $3.55 \%$ as shown in Fig. 4 (e) but still recognizable with respect to the original image.

\section{CONCLUSION}

In conclusion, we have shown that Laguerre Gaussian modes can be used to encode data onto a laser beam for transmitting information in optical systems. We were able to detect the modes in different scenarios with high fidelity. The presented technique was scarcely affected by atmospheric turbulence and no correction was applied. We are convinced that this technique will find uses in the commercial free space communication systems and in mode de-multiplexers in optical fiber.

\section{ACKNOWLEDGMENTS}

This work has been supported by the International Centre for Theoretical Physics ICTP affiliated center at the Optical Society of Tunisia and the African Laser Centre (ALC).

\section{REFERENCES}

1. Richardson, D. J., Fini, J. M., and Nelson, L. E., "Space division multiplexing in optical fibres," Nature Photonics 7, 354 (2013).

2. Chralyvy, A., "The Coming Capacity Crunch," in [35th European Conference on Optical Communication,2009. ECOC'09], Plenary paper (2009).

3. Li, G., and Liu, X., "Focus Issue: Space Multiplexed Optical Transmission," Optics Express 19, 16574-16575 (2011).

4. Chen, Y., Lobato, A., Jung, Y., Chen, H., Sleiffer, V. A. J. M., Kuschnerov, M., Fontaine, N. K., Ryf, R., Richardson, D. J., Lankl, B., and Hanik, N., "41.6 Tbit/s C-Band SDM OFDM Transmission Through 12 Spatial and Polarization Modes Over 74.17 km Few Mode Fiber," Journal of Lightwave Technology 33, 1140-1444 (2015).

5. Wang, J., Yang, J. Y., M. Fazal, I., Ahmed, N., Yan, Y., Huang, H, Ren, Y, Yue, Y, Dolinar, S, Tur, M., and Willner, A., "Terabit free-space data transmission employing orbital angular momentum multiplexing," Nature Photonics 6, 488-496 (2012).

6. Li, L., Xie, G, Yan, Y., Ren, Y., Liao, P., Ahmed, N., Zhao, Z., Bao, C., Wang, Z., Ashrafi, N., Ashrafi, S., Linquist, R., Tur, M., and Willner, A. "Experimental Demonstration of a 400-Gbit/s Free Space Optical Link Using Multiple Orbital-Angular Momentum Beams with Higher Order Radial Indices in [CLEO: Science and Innovations 2015], (2015).

7. Carpenter, J., and Wilkinson, T. D., "Characterization of Multimode Fiber by Selective Mode Excitation," Journal of Lightwave Technology 30, 1386-1392 (2012). 
8. Flamm, D., Schulze, C., Naidoo, D., Schroter, S., Forbes, A., and Duparré, M., "All-Digital Holographic Tool for Mode Excitation and Analysis in Optical Fibers," Journal of Lightwave Technology 31, 1023-1032 (2013).

9. Trichili, A., Mhlanga, T., Ismail, Y., Roux, F. S., McLaren, M., Zghal, M., and Forbes, A., "Detection of Bessel beams with digital axicons," Optics Express 22, 17553-17560 (2014).

10. Gibson, G., Courtial, J., Padgett, M., Vasnetsov, M., Pas'ko, V., Barnett, S., and Franke-Arnold, S., "Freespace information transfer using light beams carrying orbital angular momentum," Optics Express 12, 5448-5456 (2004).

11. Ren, Y., Huang, H., Xie, G., Ahmed, N., Yan, Y., I. Erkmen, B., Chandrasekaran, N., P. J. Lavery, M., K. Steinhoff, N., Tur, M., Dolinar, S., Neifeld, M., J. Padgett, M., W. Boyd, R., H. Shapiro, J., and Willner, A., "Atmospheric turbulence effects on the performance of a free space optical link employing orbital angular momentum multiplexing," Optics Letters 38, 4062-4065 (2013).

12. Xie, G., Li, L., Ren, Y., Huang, H., Yan, Y., Ahmed, N., Zhao, Z., Lavery, M. P. J., Ashrafi, N., Ashrafi, S., Bock, R., Tur, M., Molisch, A. F., and Willner, A. , "Performance metrics and design considerations for a free-space optical orbital-angular-momentummultiplexed communication link," Optica 2, 357-365 (2015).

13. Zhan, Q., "Cylindrical vector beams: from mathematical concepts to applications," Advances in Optics and Photonics 1, 1-57 (2009).

14. Siegman, A., [Lasers], University Science Books, Sausalito, California, 1986.

15. Litvin, I. A., Dudley, A., Roux, F. S., and Forbes, A. , "Azimuthal decomposition with digital holograms," Optics Express 20, 10996-11004 (2012).

16. Arrizon, V., Carrada, I. R. R., and Conzalez, L. A., "Pixelated phase computer holograms for the accurate encoding of scalar complex fields," Journal of the Optical Society of America A 24, 3500-3507 (2007).

17. Janssen, A., van Haver, S., Dirksen, P., and Braat, J., "Zernike representation and strehl ratio of optical systems with numerical aperture," Journal of Modern Optics 55, 1127-1157 (2008). 